\title{
Dietary and Other Good Health Practices for Winters
}

\author{
Aashima Gupta ${ }^{1}$, Sunita Mahajan ${ }^{2}$ and Amit Kumar Gupta ${ }^{3 *}$ \\ ${ }^{1}$ Project Assistant (Independent Social Research Project) and Scholar, DLDAV Model School, Shalimar Bagh, Delhi, India \\ ${ }^{2}$ Project Lead (Independent Social Research Project) and Former Assistant Professor, SSR College of Arts, Commerce and Science, Silvassa, \\ Dadra and Nagar Haveli (UT), India. \\ ${ }^{3}$ Freelance Consultant (Public Health) and Trainer - Clinical Excellence at HCL Healthcare India, Corporate Office, Noida, Uttar Pradesh, India. \\ *Corresponding Author: Amit Kumar Gupta, Freelance Consultant (Public Health) and Trainer - Clinical Excellence at HCL Healthcare \\ India, Corporate Office, Noida, Uttar Pradesh, India.
}

Received: September 30, 2019; Published: October 04, 2019

\begin{abstract}
To attain and maintain good health, it is important to address all components of health - preventive, promotive, curative and rehabilitative. Balanced diet for good nutrition is an essential component determining one's health. In addition, certain other good health practices can also impact health significantly. Winter is a unique season, which is also associated with various diseases and health conditions, especially among certain vulnerable populations. The present study was a short-term, social survey-based descriptive research in which focus group discussions were held with select physicians and dieticians; and a thematic analysis of the observations was done to understand the current dietary and other good health practices that should be followed during winters. Key dietary and other good health practices for winters, as inferred from this study, have been discussed here.

Keywords: Diet; Health; Nutrition; Practices; Winter
\end{abstract}

\section{Abbreviations}

FGD: Focus Group Discussion; WHO: World Health Organization

\section{Introduction}

The World Health Organization (WHO) defined health in its broader sense as "a state of complete physical, mental, and social well-being and not merely the absence of disease or infirmity" [1]. It is important to address all components of health - preventive, promotive, curative and rehabilitative - to attain such a well-being. Various social and other determinants of health, particularly the health-promoting factors found in our living and working conditions, influence health at individual and community levels [2]

A diet is all that one consumes in a day. Nutrition is an essential component determining one's health as a healthy diet helps maintain and/or improve overall health. A balanced diet is the diet containing adequate quantities and proportions of various nutrients required for the body [3]. In addition to nutrition, adequate awareness about other health practices and healthy socio-cultural practices also impact our health significantly [4].

Winter is the coldest season of the year. Many diseases and health conditions tend to occur or tend to occur more frequently during winters (such as cough and cold, skin dryness and eczema, heart attacks, asthma attacks, road traffic accidents, etc.) [5]. Therefore, it is inevitable that everyone should know about key good health practices in order to either prevent such winter- related diseases, or at least, take timely action for management of such condition. The present study aimed to understand the current dietary and other good health practices that should be followed during winters.

\section{Materials and Methods}

The present study was conducted as a short-term, social survey-based descriptive research aimed to understand the current dietary and other good health practices that should be followed during winters. Its methodology involved focus group discussion (FGD) [6]. A sample of technical experts on health (medical doctors) and dietetics (dieticians) dealing with members of the community were selected from the available resources in the vicinity of the study area. The FGDs were held in two groups - the first group comprised 10 clinicians (8 general physicians and 2 internal medicine physicians) and the second group comprised 5 dieticians. During each FGD, the subject matter of health in winters and healthy practices for winters was discussed, focusing on dietary and other good health practices that should be followed during winters; the key points of the discussion were noted under various themes; and a thematic analysis of these observations was done and discussed to draw inferences.

\section{Results and Discussion}

Observations of the present study suggested that appropriate health awareness, certain health promotion activities and specific health measures could be very useful to maintain health and 
prevent health issues during winters. Diet, healthy practices, and a proactive approach to manage health issues well in time were found to be the major themes of consideration and discussion during the FGDs. The FGD with physicians focused on preventive and promotive aspects of health and early referral for medical support; on the other hand, the FGD with dieticians focused on balanced diet and other healthy dietary practices to maintain good health during winters.

Key dietary and other good health practices for winters, as per the observations of this study, are discussed in brief below:

- $\quad$ Eat healthy: Eat fruits and vegetables which pack nutrients and help lower the risk for certain diseases. Limit your portion sizes and foods high in fat, salt and sugar. Although energy-rich (calorie-rich) food items are perceived to be taken in larger quantities during the winter, it is the balanced diet that is required to fulfill body's need in this season too [7].

- Drink adequate water and other liquids: Keep yourself well-hydrated. So, drink plenty of water and other liquids throughout the day. Some people neglect water intake during winter due to their misconceptions about importance of water intake, not feeling thirsty in cold weather, and avoiding frequent water intake so as not to go for voiding too often. Adequate intake of water and other liquids (around 2-3 L/day for an adult) keeps the skin well-hydrated, healthy, and less prone to dryness, eczema and similar other skin problems common during winters [8].

- Prepare and store foods safely: Before handling food items and eatables, one must wash his/her hands and the surfaces (very important for cooks and housewives). Avoid cross-contamination at the kitchen area. Foods should be cooked to proper temperatures; and they should be stored properly or refrigerated promptly [9].

- Celebrate responsibly: Everyone tends to relish on junk foods and unhealthy beverages during birthdays, festivities and similar other occasions. There can be various healthy yet tastier food choices during such good times. Make a change and showcase your healthy cuisines and food items to promote health during winters [10]. Deepawali and New Year seasons see a lot drunk driving. Everyone must remember not to drink and drive. One should also not let others drink and drive. Whenever people drive drunk, they put everyone on the road in danger. Choose not to drink and drive and help others do the same [11].

- $\quad$ Exercise and stay active: Many people tend to stay away from doing exercise during winters. One can choose indoor options in case it is very chilly or foggy outside. During winters also, remain active by doing some physical activity such as morning or evening walk, jogging, yoga etc. Exercise for at least 150 minutes a week (choose either 30 minutes daily for 5 days in a week, or 50 minutes alternate day for 3 days in a week, or make your own customized schedule) [12]. Help kids and teens also to be physically active through such exercises.

- $\quad$ Prevent spread of germs: Winter is also the flu season and many-a-times such flu or other infections can complicate to result into serious health issues. One should wash hands often to help prevent the spread of germs. Wash your hands with soap and clean running water for 20-30 seconds [13].

- Maintain the warmth: The cold weather of winters and associated fogginess of the climate can affect anybody, but more commonly the vulnerable individuals such as the elderly, the kids, and those with chronic respiratory \& other diseases (like asthma, heart disease, diabetes etc.). They should wear appropriate outdoor clothing (viz. light, warm layers, gloves, scarves, sweaters and jackets). Bundle up to stay dry and warm to shun winter-related health problems [14].

- Manage stress: If you are feeling stressed out, overwhelmed, or out of control, do not ignore. Give yourself a break and destress yourself. Some of the best ways to manage stress are to find support, connect socially, and get plenty of sleep. Have some "Me Time" during which you can do the things and activities you like (such as listening to music, dancing, doing your hobby, or doing just nothing!) [15].

- Be smoke-free and dust-free: Smoking and secondhand smoke harm the respiratory system; so, both should be avoided. Smokers have greater health risks during winter because of their smoking acts; but nonsmokers also are at risk when exposed to smoke. Also, avoid going outside in poor-quality air (like areas with fog, dust, smog, excessive vehicular smoke etc.) [16].

- $\quad$ Practice fire safety: During winter, we use heaters at our home - a potential fire hazard; so most residential fires occur during the winter months. Don't leave space heaters, or food being cooked on stoves, or burning candles unattended. One should have an emergency plan and should practice it regularly [17].

- Care of eyes: The cold, dry air in winter and the dryness due to use of heaters can lead to dryness of eyes (causing burning, itching and a feeling of grittiness in eyes) and airways (causing chest tightness, cough, breathlessness and aggravation of an underlying respiratory disease). Maintain adequate moisture in the air (one can use humidifier or simply keep a watercontaining bowl in room while using heater). Drink plenty of water and hot beverages. Steam inhalation and use of artificial tears also help. One should not delay seeking medical help in case of any such relevant symptoms [18].

- Skin care: Skin dryness and skin cracks can happen in winter. One should maintain good hydration and take balanced diet. Taking short showers, using humidifiers, and applying skin 
moisturizer also help in skin care [19]. In case of need, do not hesitate to seek help of an expert (physician or dietician).

- Vaccination: Vaccinations help prevent diseases and save lives. Certain diseases (such as influenza, acute exacerbation of chronic bronchitis, etc.), which occur more frequently during winters, can be prevented significantly through annual administration of flu vaccine prior to the winter season. Few other vaccines are also useful for protection during winter season [20]. So, one should discuss with his/her family physician for further information and necessary advice regarding vaccination for this purpose.

- $\quad$ Extra care of the vulnerable ones: Young children, the elderly and persons with certain chronic health conditions (like heart disease, asthma, chronic bronchitis etc.) have higher risk of health problems and complications during winter season [21]. They require extra care for protection against harmful effects of the cold weather and may need prompt management of any health issue or acute episode. One must never delay in seeking help in case someone out of these vulnerable people is having any symptom or health issue.

\section{Conclusion}

Although this was a small study conducted as a short-term project, its observations and inferences are significant from the social health perspective. Winter do make a unique season for us - cold weather, snow games, woolens, heating appliances, hot beverages \& other delicacies. However, it also brings along various health concerns, for which one needs to take some extra care, especially for kids, elderly and other vulnerable persons. Balanced diet and other good health practices can help maintain healthy body and mind during winters. Larger, prospective studies can throw more light on to such healthy practices and their exact effects on overall health.

\section{Conflict of Interest}

None. There is no direct or indirect real or perceived financial interests or conflicts; and this work of the authors is without any prejudice to their academic or professional associations.

\section{Bibliography}

1. World Health Organization. "Health for All, Sr. No.1". Geneva: World Health Organization (1978).

2. World Health Organization. "Closing the Gap in a Generation: Health Equity Through Action on the Social Determinants of Health". Commission on Social Determinants of Health, Geneva: World Health Organization (2008).

3. Lean Michael EJ. "Principles of Human Nutrition". Medicine 43.2 (2015): 61-65.

4. Healthshare. "Why spreading health awareness is important". (2017).
5. Wikipedia contributors. "Winter". Wikipedia, The Free Encyclopedia, Wikipedia, The Free Encyclopedia.

6. Prasad Monishkumar and Christine Garcia. "How to conduct a successful focus group discussion” (2017).

7. Whiting Kate. "Healthy winter diet: The best foods to eat to stay well this winter" (2017).

8. Tamlin, Stephen. "5 Major Reasons Why You Should Drink More Water Over Winter" (2017).

9. University of Nebraska-Lincoln. "Helpful Winter Holiday Food Preparation, Safety and Healthy Eating" (2017).

10. Faaiuaso, Elecia. "Healthy Celebration Toolkit". Bronx Health Reach, The Institute for Family Health (2019).

11. Williams Ashley. "How drinking alcohol makes you vulnerable in cold weather". AccuWeather (2019).

12. American Heart Association. "How to Stay Active in Cold Weather". AccuWeather (2019).

13. WebMD. "Stop the Spread of Cold and Flu Germs". (2019).

14. Green, Amanda. "12 Toasty Tips for Staying Warm in Cold Weather” (2019).

15. Stinson Annakeara. "Does Cold Weather Cause Stress? 6 Ways To Relieve Anxiety In The Winter". Elite Daily (2019).

16. Wikipedia contributors. "Air Pollution in India". Wikipedia, The Free Encyclopedia, Wikipedia, The Free Encyclopedia (2019).

17. National Fire Protection Association. "Put A Freeze on Winter Fires" (2019).

18. Sightsavers India. “Winter Eye Care Tips” (2019).

19. Marks Hedy. “Top 10 Tips for Healthy Winter Skin” (2018).

20. Brandywine Senior Living. "5 Vaccines That Your Loved One Should Have In Order To Stay Healthy This Winter" (2019).

21. World Health Organization. "WHO warns: stay alert and help vulnerable as cold grips Europe". Geneva: World Health Organization (2018).

Volume 3 Issue 11 November 2019

(c) All rights are reserved by Aashima Gupta., et al. 